\title{
Glutamate reduces glucose utilization while concomitantly enhancing AQP9 and MCT2 expression in cultured rat hippocampal neurons
}

\author{
Fabio Tescarollo ${ }^{1,2}$, Luciene Covolan ${ }^{1}$ and Luc Pellerin ${ }^{2 *}$ \\ 1 Departamento de Fisiologia, Universidade Federal de São Paulo, São Paulo, Brazil \\ ${ }^{2}$ Laboratory of Neuroenergetics, Department of Physiology, University of Lausanne, Lausanne, Switzerland
}

Edited by:

Shaida A. Andrabi, Johns Hopkins

University School of Medicine, USA

Reviewed by:

Anne-Karine Bouzier-Sore,

CNRS-/Université Victor Segalen,

France

Angus M. Brown, University of Nottingham, UK

Shaida A. Andrabi, Johns Hopkins

University School of Medicine, USA

\section{*Correspondence:}

Luc Pellerin, Department of Physiology, 7 Rue du Bugnon, 1005 Lausanne, Switzerland e-mail: luc.pellerin@unil.ch
The excitatory neurotransmitter glutamate has been reported to have a major impact on brain energy metabolism. Using primary cultures of rat hippocampal neurons, we observed that glutamate reduces glucose utilization in this cell type, suggesting alteration in mitochondrial oxidative metabolism. The aquaglyceroporin AOP9 and the monocarboxylate transporter MCT2, two transporters for oxidative energy substrates, appear to be present in mitochondria of these neurons. Moreover, they not only co-localize but they interact with each other as they were found to co-immunoprecipitate from hippocampal neuron homogenates. Exposure of cultured hippocampal neurons to glutamate $100 \mu \mathrm{M}$ for $1 \mathrm{~h}$ led to enhanced expression of both AOP9 and MCT2 at the protein level without any significant change at the mRNA level. In parallel, a similar increase in the protein expression of LDHA was evidenced without an effect on the mRNA level. These data suggest that glutamate exerts an influence on neuronal energy metabolism likely through a regulation of the expression of some key mitochondrial proteins.

\section{Keywords: brain energy metabolism, mitochondria, lactate dehydrogenase, monocarboxylate transporter,} aquaglyceroporin

\section{INTRODUCTION}

The human brain represents only $2 \%$ of the total body weight but it accounts for a much larger proportion of the total energy expenditures of the organism (Magistretti, 1999). Under physiological conditions, glucose is considered the major energy substrate taken up and used by the adult brain to supply its energy needs. A linear correlation between glucose utilization and brain activity has been reported for various brain regions in different conditions (Sokoloff, 1993). This tight relationship has formed the basis for the development of some functional brain imaging techniques (e.g., FDG-PET) to visualize changes in brain activity during cognitive tasks. Despite these important developments, the specific fate of glucose at the cellular and molecular levels remains elusive.

Glutamatergic synapses are by far the predominant type of synapses in the central nervous system and constitute the main source of excitatory neurotransmission. Glutamate enhances both glucose transport (Loaiza et al., 2003) as well as uptake and utilization (Pellerin and Magistretti, 1994, 1996a) in cultured astrocytes. As a consequence, a major part of glucose uptake taking place in an activated brain region and visualized by functional brain imaging is due to astrocytic utilization (Pellerin and Magistretti, 1996b, 2012; Figley and Stroman, 2011). Such a view is reinforced by recent observations made ex vivo and in vivo. First, it was demonstrated in cerebellar slices using fluorescent glucose analogs that both glucose transport and metabolism occur preferentially in Bergmann glia over Purkinje cells while the same observation was made for astrocytes over neurons in hippocampal slices (Barros et al., 2009; Jakoby et al., 2014). In addition, it was shown in experiments performed in anesthetized rats that whisker stimulation caused a significant increase of glucose uptake in astrocytes, but not in neurons, in the activated somatosensory cortex (Chuquet et al., 2010). While these data indicate that a prominent part of glucose utilization take place in astrocytes, they also suggest that active neurons must obtain an alternative energy substrate to cover their enhanced energy needs.

In addition to glucose, neurons are able to use a few alternative oxidative energy substrates that include monocarboxylates such as lactate or the ketone bodies (Pellerin, 2010). The use of these energy substrates by brain cells requires the presence of specific transporters (Pierre and Pellerin, 2005). Indeed, neurons predominantly express the high affinity monocarboxylate transporter MCT2 (Bergersen et al., 2001; Pierre et al., 2002). In addition, at least some populations of neurons also express the aquaglyceroporin AQP9 (Badaut, 2010), a carrier known to allow the passage not only of glycerol but also of lactate (Badaut et al., 2007). So far, few informations exist about the relative subcellular distribution of these two transporters in neurons as well as the possible regulation of their expression in relation with glutamatergic neurotransmission. In this study, we aimed to determine (1) whether glutamate modulates glucose utilization in rat hippocampal neurons, (2) if AQP9 and MCT2 can be coexpressed by neuronal mitochondria, and (3) if glutamate could alter the expression of AQP9 and/or MCT2 in the same neuronal population. 


\section{MATERIALS AND METHODS HIPPOCAMPAL NEURONAL CULTURES}

All animal experiments were performed in accordance with the Swiss animal welfare laws under the authorization $\mathrm{n}^{\circ}$ VD 1251.4 delivered by the Service de la consommation et des affaires vétérinaires du Canton de Vaud, Switzerland. Hippocampi were carefully dissected from E18 Wistar rat embryos and then the cells were placed in $0.25 \%$ Tripsin-EDTA buffer supplemented with $100 \mathrm{mM}$ of DNAse I. In order to stop the effect of trypsin, neurobasal culture medium with $1 \%$ fetal bovine serum was added and then cells were mechanically dissociated by gentle passage through fire-polished glass pipets. After centrifugation $(5 \mathrm{~min}$, $300 \mathrm{~g}$ ), pellets of dissociated cells were resuspended in neurobasal culture medium supplemented with B27 (2\%), L-glutamine $(0.5 \mathrm{mM})$ and an antibiotic cocktail (Neugene, $0.5 \%)$. Viable cells were counted using a Scepter Handheld Automatic Cell Counter (Millipore, Billerica, USA). Cells were plated at a density of $1.5 \times 10^{5}$ cells/well on Poly-D-Lysine coated coverslips in 24-well dishes containing $0.5 \mathrm{~mL}$ of culture medium for immunofluorescence experiments and $7.5 \times 10^{5}$ cells/well in Poly-D-Lysine coated 6-well dishes containing $2 \mathrm{ml}$ of culture medium for the other assays. Cells were maintained at $37^{\circ} \mathrm{C}$ in a $5 \% \mathrm{CO}_{2}$ atmosphere, without any further changes.

\section{CELL CULTURE STIMULATION PROCEDURES}

After 6 days in vitro (DIV), cultured neurons were stimulated by treatment with $100 \mu \mathrm{M}$ of L-glutamic acid for $1 \mathrm{~h}$ (Bliss et al., 2004; Hartz et al., 2009). This treatment was used to mimic the increased neuronal excitability that occurs during an ictal epileptic episode (Churn et al., 1991; Blair et al., 2008; Hartz et al., 2009).

\section{[ $\left.{ }^{3} \mathrm{H}\right] 2 \mathrm{DG}$ UPTAKE AND LACTATE RELEASE MEASUREMENT}

Primary cultures of rat hippocampal neurons were used at confluence, usually between 6 and 7 days after seeding. 2-Deoxy-D$\left[1,2-{ }^{3} \mathrm{H}\right]$ glucose $\left(\left[{ }^{3} \mathrm{H}\right] 2 \mathrm{DG}\right)$ uptake was determined as described previously (Pellerin and Magistretti, 1994). On the day of the experiment, the culture medium was replaced by serum-free DMEM supplemented with $5 \mathrm{mM}$ glucose, $44 \mathrm{mM} \mathrm{NaHCO}_{3}$, $0.06 \mathrm{~g} / \mathrm{L}$ penicillin, $0.1 \mathrm{~g} / \mathrm{L}$ streptomycin, and $0.045 \mathrm{mM}$ phenol red $\left(\mathrm{DMEM}_{5}\right)$. Cultured hippocampal neurons were incubated for $2 \mathrm{~h}$ at $37^{\circ} \mathrm{C}$ in a water-saturated atmosphere containing 5\% $\mathrm{CO}_{2} / 95 \%$ air. The medium was then replaced by $2 \mathrm{ml}$ of the same $\mathrm{DMEM}_{5}$ medium containing $\left[{ }^{3} \mathrm{H}\right] 2 \mathrm{DG}$ at a concentration of $1 \mu \mathrm{Ci} / \mathrm{ml}(33 \mathrm{nM})$. In order to study the effect of stimulation in cultured cells, $100 \mu \mathrm{M}$ of glutamate were added to the medium $1 \mathrm{~h}$ before substitution by the medium containing $\left[{ }^{3} \mathrm{H}\right] 2 \mathrm{DG}$ as well as $100 \mu \mathrm{M}$ glutamate and the cells were further incubated for $20 \mathrm{~min}$ in the same conditions as previously indicated. The reaction was stopped by aspiration of the medium followed by rinsing the cells three times with ice-cold phosphate buffered saline and $0.1 \mathrm{M} \mathrm{NaOH} / 0.1 \%$ Triton $\mathrm{X}-100$ was added to lyse the cells. Aliquots of $500 \mu \mathrm{l}$ were assayed for radioactivity by liquid scintillation counting, while $50 \mu \mathrm{l}$ aliquots were used for measurement of protein by the method of Bradford (1976). Results, which represent glucose transporter-mediated uptake and subsequent phosphorylation, were calculated by subtracting from total counts the portion that was not inhibited by the glucose transporter inhibitor cytochalasin B $(10 \mu \mathrm{M})$. The cytochalasin-sensitive uptake accounted for $80 \%$ of total uptake. Lactate measurement was performed as previously described (Pellerin and Magistretti, 1994). Briefly, supernatants from 2DG uptake experiments were collected to determine lactate production and release. Two $100 \mu \mathrm{l}$ aliquots for each supernatant were placed in separate wells of a 96-well plate. To each well was added $100 \mu \mathrm{l}$ of a solution containing lactate dehydrogenase $70 \mathrm{U} / \mathrm{ml}$ (Sigma, Buchs, Switzerland) and NAD $15 \mathrm{mM}$ (Acros organics, Geel, Belgium) in glycine-semicarbazide buffer $0.33 \mathrm{M}, \mathrm{pH} 10$. After 1 hour at $37^{\circ} \mathrm{C}$, plates were read at $340 \mathrm{~nm}$ to detect production of NADH. A standard curve with known concentrations of lactate was used to determine lactate concentrations, corrected for the amount of protein in the same culture well.

\section{MITOTRACKER STAINING}

The red Mitotracker ${ }^{\circledR}$ (Molecular Probes, Eugene, USA), a mitochondrial potential-sensitive dye, was added to the culture medium of cultured hippocampal neurons at 6 DIV at a concentration of $150 \mathrm{nM}$ for $30 \mathrm{~min}$ at $37^{\circ} \mathrm{C}$. Samples were washed with PBS and fixed in $4 \%$ paraformaldehyde for further use in immunolabeling and confocal fluorescence experiments.

\section{ANTIBODIES}

The commercial antibodies used in this work were anti-AQP9 (Santa Cruz Biotechnology, California, USA), anti-lactate dehydrogenase A, (Abcam, Cambridge, UK), and anti- $\beta$-tubulin (Sigma, Buchs, Switzerland). The polyclonal anti-MCT2 antibody is a previously characterized homemade antibody (Pierre et al., 2000).

\section{IMMUNOCYTOCHEMISTRY}

Rat hippocampal neurons grown directly on coverslips were used to perform immunofluorescence experiments. After removal of the culture medium, cells were carefully rinsed in phosphatebuffered saline (PBS) at $37^{\circ} \mathrm{C}$ and directly postfixed in an icecold paraformaldehyde fixative (PFA $4 \%$ in PBS for $30 \mathrm{~min}$ at $\left.20^{\circ} \mathrm{C}\right)$. Fixed cells were treated with casein $(0.5 \%$ in $\mathrm{PBS})$ for $1 \mathrm{~h}$ at room temperature to block non-specific sites. For immunostaining, cultures were incubated overnight at $4^{\circ} \mathrm{C}$ in $50 \mu \mathrm{L}$ of freshly prepared anti-MCT2 antibody solution [antiMCT2 diluted 1:50 (Pierre et al., 2000) in PBS containing 0.25\% bovine serum albumin] and/or with anti-AQP9 diluted 1:5 in PBS containing $0.25 \%$ bovine serum albumin. After carefully rinsing in PBS, cultures were incubated for $2 \mathrm{~h}$ at room temperature in a solution containing Cy3-conjugated anti-rabbit IgG or FITC-conjugated anti-goat IgG (diluted 1:250; Jackson Immunoresearch, Baltimore, USA). After rinsing in PBS twice and a final rinsing in water, coverslips were mounted with Vectashield containing DAPI (Reactolab SA, Burlingame, CA, USA). Coverslips containing the cells were examined and photographed using a LSM 710 Quasar Confocal Microscope (Zeiss, Hallbergmoos, Germany). Images were obtained using a Plan Apochromat $63 \times$ objective and acquired using a cooled CCD camera (Axiocam, Zeiss, Hallbergmoos, Germany), together with the Zeiss 2009 software (Zeiss, Hallbergmoos, Germany). Five 
neurons were randomly chosen on each coverslip. Pictures were then analyzed using the plugin WCIF-ImageJ for co-localization analysis for ImageJ NIH software (National Institutes of Health Image program, version 1.62, Rockville Pike, USA). Images were converted to 8-bit images for each red and green label of each image, and then the level of co-localization was determined by Mander's overlap coefficient, and the data are presented as percentage of co-localization.

\section{ELECTROPHORESIS AND WESTERN BLOTTING}

Rat hippocampal neurons in each culture dish were homogenized in $500 \mu \mathrm{L}$ of lysis buffer containing the following: Tris$\mathrm{HCl}, \mathrm{pH}$ 6.8, $80 \mathrm{mmol} / \mathrm{L}$; EDTA (ethylene diamine tetraacetic acid), $5 \mathrm{mmol} / \mathrm{L}$; NP-40, $1 \%$ or SDS, $5 \%$ and a mixture of protease inhibitors (Complete, Roche Molecular Biochemicals, Mannheim, Germany). Control and each stimulated condition were performed in triplicate. Protein samples from rat hippocampal neuronal cultures were sonicated and heated at $95^{\circ} \mathrm{C}$ for $5 \mathrm{~min}$ in half the final volume of SDS-PAGE sample buffer (Tris$\mathrm{HCl}, 62.5 \mathrm{mmol} / \mathrm{L}$; DTT, $50 \mathrm{mmol} / \mathrm{L}$; SDS, 2\%; glycerol, 10\%; and bromophenol blue, $0.1 \%$ ). Samples were loaded onto polyacrylamide gels composed of a $4.5 \%$ acrylamide-bisacrylamide stacking gel and a $12 \%$ acrylamide-bisacrylamide running gel. After electrophoresis, proteins were transferred onto nitrocellulose membranes (Trans-Blot Transfer Medium 162-0115; BioRad, Reinach, Switzerland) using a Transblot semi-dry transfer cell (Bio-Rad, Reinach, Switzerland). For protein detection, membranes were incubated in a blocking solution of Tris-buffered saline supplemented with Tween-20 (TBST; Tris-HCl, pH 7.5, $50 \mathrm{mmol} / \mathrm{L} ; \mathrm{NaCl}, 150 \mathrm{mmol} / \mathrm{L}$; and Tween-20, 0.1\%) containing $5 \%$ non-fat milk for $1 \mathrm{~h}$ at room temperature. Membranes were incubated overnight at $4^{\circ} \mathrm{C}$ in a solution containing the primary antibody. After washing in TBST, membranes were incubated with horseradish peroxidase (HRP) conjugated secondary antibodies in TBST containing 1\% of non-fat milk, for $2 \mathrm{~h}$ at room temperature. After washing in TBST, membranes were submitted to a chemiluminescent reaction using ECL (GE Healthcare, Ecublens, Switzerland), and then photographed using the ChemiDoc XRS + (BioRad). Quantification was made using ImageJ software (NIH, Maryland, USA).

\section{IMMUNOPRECIPITATION}

Primary cultures of rat hippocampal neurons were collected and homogenized in ice-cold lysis buffer containing $20 \mathrm{mM}$ Tris $\mathrm{HCl}$; $137 \mathrm{mM} \mathrm{NaCl} ; 10 \%$ Glycerol; 1\% n-octyl-b-D-glucoside (OG) and $2 \mathrm{mM}$ EDTA, $\mathrm{pH} 7.4$, in presence of a complete set of protease inhibitors (Complete; Roche Molecular Biochemicals, Mannheim, Germany) and the homogenate was sonicated three times for $5 \mathrm{~s}$ each. The homogenate was reacted with the primary antibody (1:50 anti-MCT2) overnight at $4^{\circ} \mathrm{C}$. Agarose-conjugated protein $\mathrm{A}$ was added and incubated with gentle rocking at $4^{\circ} \mathrm{C}$ for $2 \mathrm{~h}$. This complex was then centrifuged at $14,000 \mathrm{~g}$ during $30 \mathrm{~s}$ at $4^{\circ} \mathrm{C}$ and washed five times with ice-cold lysis buffer. The pellet was then resuspended in $3 \times$ SDS sample buffer and microcentrifuged for $30 \mathrm{~s}$. The sample was heated to $95-100^{\circ} \mathrm{C}$ for $5 \mathrm{~min}$ and centrifuged for $1 \mathrm{~min}$ at $14,000 \mathrm{~g}$. The supernatant was collected, loaded on SDS-PAGE and Western blot was performed as described above to verify the presence of AQP9 in the MCT2 immunoprecipitate. Total protein extract from primary cultures of rat hippocampal neurons was loaded as control for each experiment.

\section{QUANTITATIVE REAL-TIME POLYMERASE CHAIN REACTION}

Quantitative determination of AQP9, MCT2, and LDHA mRNA expression levels was performed by quantitative Real-Time Polymerase Chain Reaction (qRT-PCR) according to Heid et al. (1996) using a StepOnePlus or ViiA 7 sequence detection system from Applied Biosystems (Rotkreuz, Switzerland). The following sets of oligonucleotides (Microsynth, Balgach, Switzerland) were used: ActinFo, CTTCTTTGCAGCTCCTTCGT; ActinRe, ATATCGTCATCCATGGCGAAC (Embl: X03672); MCT2Fo, CA GCAACAGCGTGATAGAGCTT; MCT2Re, TGGTTGCAGGTTG AATGCTAAT (Embl: NM_017302); AQP9Fo, GATGGACTCA

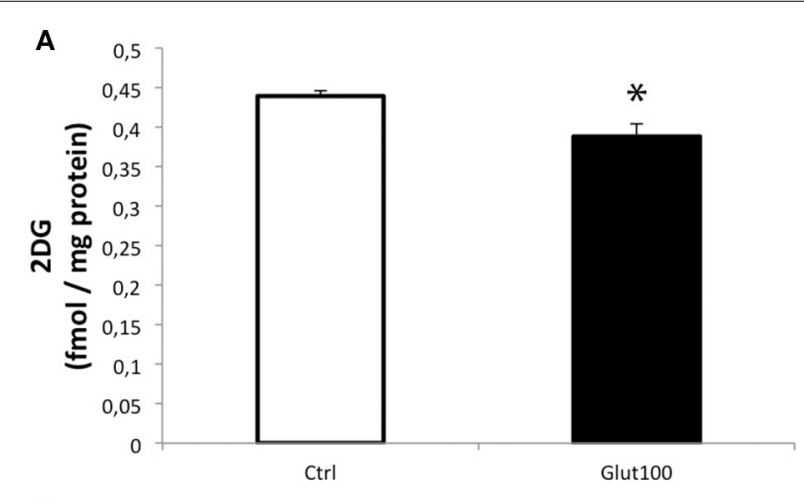

B

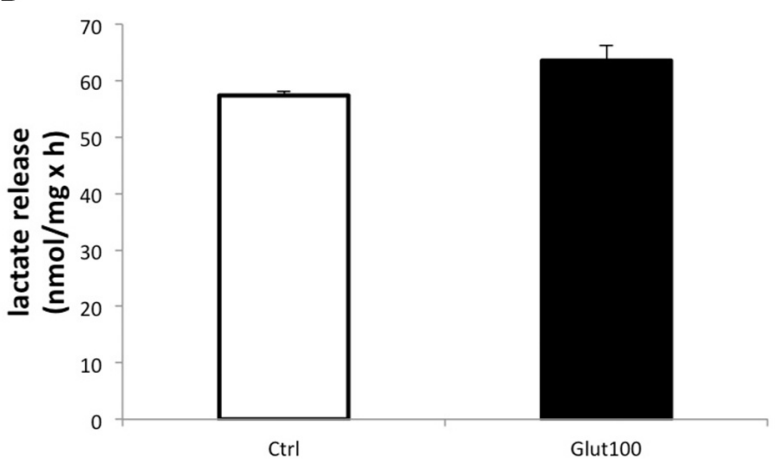

FIGURE 1 | Effect of glutamate on glucose utilization and lactate production in cultured rat hippocampal neurons. (A)

$\left[{ }^{3} \mathrm{H}\right]$-2-deoxyglucose uptake and accumulation was measured in primary cultures of rat hippocampal neurons for 20 min either without exposure to glutamate (Ctrl) or with prior treatment with glutamate $100 \mu \mathrm{M}$ for $1 \mathrm{~h}$ followed by the presence of the same concentration of glutamate during the $\left[{ }^{3} \mathrm{H}\right] 2 \mathrm{DG}$ uptake measurement (Glut100). (B) Lactate production and release was measured in primary cultures of rat hippocampal neurons for 20 min either without exposure to glutamate (Ctrl) or with prior treatment with glutamate $100 \mu \mathrm{M}$ for $1 \mathrm{~h}$ followed by the presence of the same concentration of glutamate during the lactate production measurement (Glut100). Data represent mean $\pm \mathrm{SD}$ with $n=3$. Statistical analysis was performed using a non-parametric Mann-Whitney test. An asterisk indicates 2DG uptake significantly different from control with $p<0.05$. 2DG, 2-Deoxyglucose; CTRL, Control. 
TGGCCTTTGCTG; AQP9Re, CAATCATAGGACCCACGACA GG (Embl: NM_022960.2); LDHAFo, TGGCCTCTCCGTGG CAGACT; LDHARe, CCCCCAGACCACCTCAACACAA (Embl: NM_017025.1).

\section{STATISTICAL ANALYSIS}

Experimental data are expressed as mean \pm standard deviation (SD). They were statistically analyzed with a non-parametric Mann-Whitney test. Statistical significance was set at $p<0.05$.

\section{RESULTS}

Glucose utilization of cultured hippocampal neurons in response to glutamate stimulation was evaluated by measuring ${ }^{3} \mathrm{H}$-2-deoxyglucose (2DG) uptake. Treatment of cultured hippocampal neurons with glutamate $100 \mu \mathrm{M}$ for $1 \mathrm{~h}$ induced a significant decrease in 2DG uptake $(-11.74 \pm 3.7 \%)$ compared to the control group (Figure 1A). In parallel, measurement of lactate accumulated in the medium did not reveal any significant difference, arguing against a reduction in glycolytic flux ending with lactate production (Figure 1B). Such an unexpected effect of glutamate on glucose utilization in neurons suggested that some modifications, notably on oxidative metabolism, might take place in these cells following an exposure to glutamate. For this reason, the expression of the aquaglyceroporin AQP9 and the monocarboxylate transporter MCT2 which are involved in the transport of oxidative substrates was investigated in this preparation.
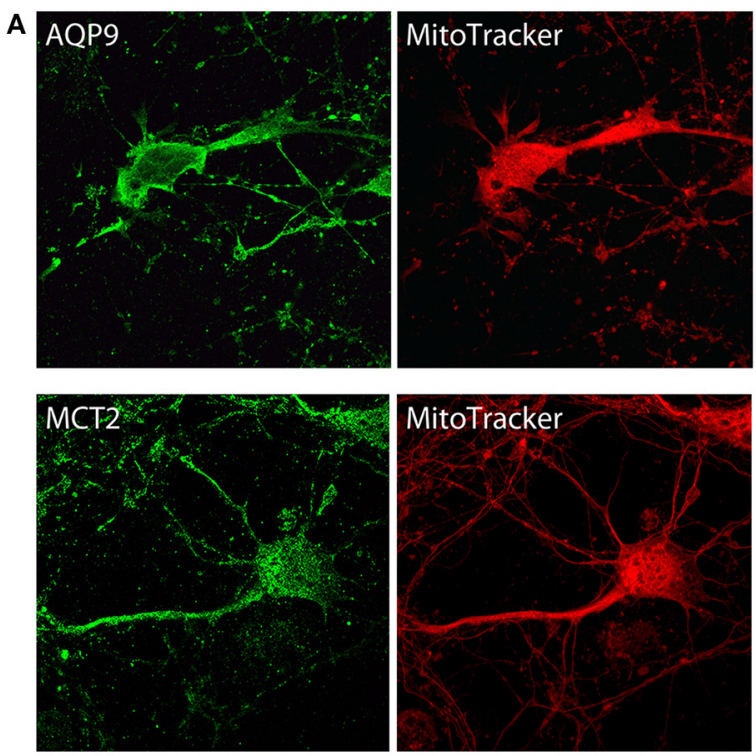

B

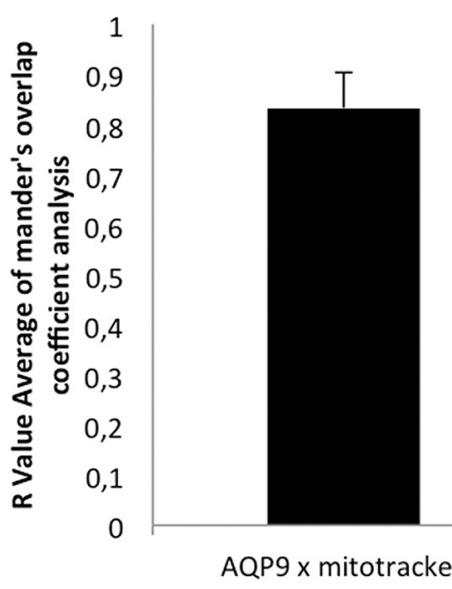

FIGURE 2 | Immunofluorescent localization of AQP9 and MCT2 in mitochondria of cultured rat hippocampal neurons (A) Immuno fluorescent labelings for AQP9 (in green, upper row) or MCT2 (in green, bottom row) and mitotracker staining (in red) in cultured rat hippocampal neurons. Colocalization between AQP9 or MCT2 and mitotracker appears as yellow on merged images (Merge). Nuclei (in blue)
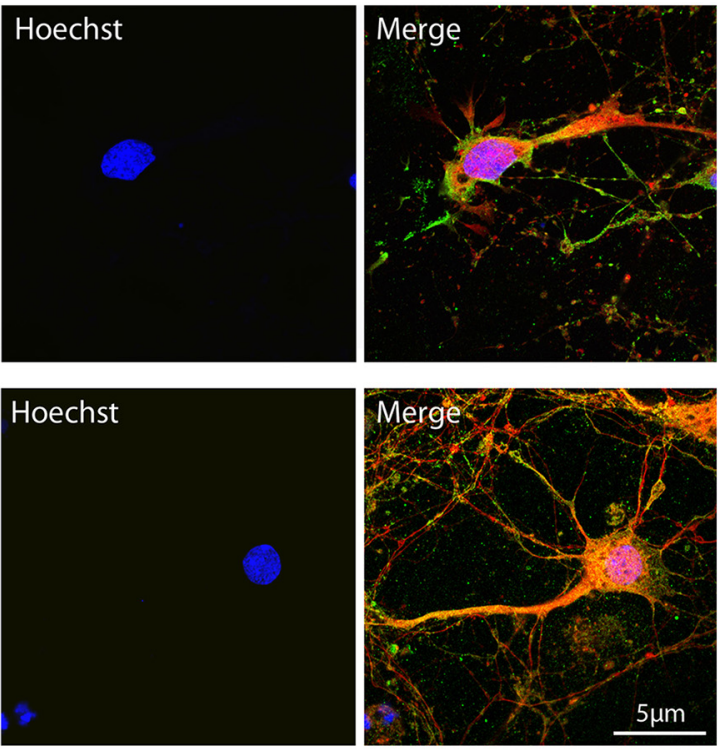

were labeled using Hoechst staining. (B) Quantification of the degree of colocalization between AQP9 or MCT2 and mitotracker. The degree of colocalization was assessed with Mander's overlap coefficient obtained by using the plugin WCIF-ImageJ for colocalization analysis for Image $\mathrm{NIH}$ software. Immunofluorescence was visualized using confocal microscopy with appropriate filters. Data represent mean \pm SD with $n=5$. 
Double labelings with a mitochondrial marker, the MitoTracker ${ }^{\circledR}$, and either MCT2 or AQP9 were performed on cultured hippocampal neurons and analyzed by confocal microscopy (Figure 2). Observation of fluorescence images revealed that co-localization was present for both AQP9 and MCT2 (Figure 2A). Quantification of the degree of colocalization revealed that it reaches $83.2 \pm 7.1 \%$ between AQP9 and the mitochondrial marker while it was of $85.2 \pm 6.2 \%$ for MCT2 and the same mitochondrial marker (Figure 2B).

Such a similar degree of co-localization with the same mitochondrial marker suggested that AQP9 and MCT2 could colocalize together. Indeed, using double immunofluorescence labeling and confocal microscopy, it could be observed that AQP9 and MCT2 shared a similar subcellular distribution in cultured hippocampal neurons (Figure 3A). Quantification of the level of co-localization between MCT2 and AQP9 staining in these neurons yielded a value of $83.0 \pm 4.5 \%$. Such a high degree of co-localization raised the possibility of a closer interaction between the two proteins. In order to determine whether MCT2 interacts with AQP9 protein, MCT2 was immunoprecipitated from total homogenates of cultured hippocampal cells, protein fractions obtained from MCT2 immunoprecipitates were separated by electrophoresis and analyzed for the presence of AQP9 by western blotting. Results show that AQP9 is indeed detected in the MCT2 immunoprecipitate (Figure 3B), indicating that AQP9 and MCT2 are linked to each other.

The possibility that the level of expression of these two energetic substrate transporters could be regulated by glutamate has been investigated in rat hippocampal cultured neurons. In parallel, the expression of the isoform A of lactate dehydrogenase, a key enzyme for the use of lactate as oxidative substrate, was also determined. First, exposure of cultured hippocampal neurons to glutamate $(100 \mu \mathrm{M})$ for $1 \mathrm{~h}$ caused no significant change in the expression level of either AQP9, MCT2, or LDHA (Figure 4A). A similar analysis was performed at the protein level. In this case, it could be observed by Western blot that AQP9, MCT2, and LDHA protein expression was enhanced in cultured hippocampal neurons after exposure for $1 \mathrm{~h}$ to glutamate $100 \mu \mathrm{M}$ (Figure 4B, upper panel). Quantification of the level of expression revealed increases of $25.3 \pm 1.46 \%$ (AQP9), $29.9 \pm 1.98 \%$ (MCT2), and $50.33 \pm 3.93 \%$ (LDHA) when compared to control.

\section{DISCUSSION}

Glutamate is the main excitatory neurotransmitter in the central nervous system. It is purported that its depolarizing action on neurons should lead to increased energy demands in this cell

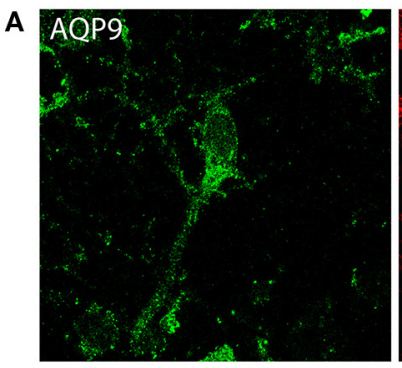

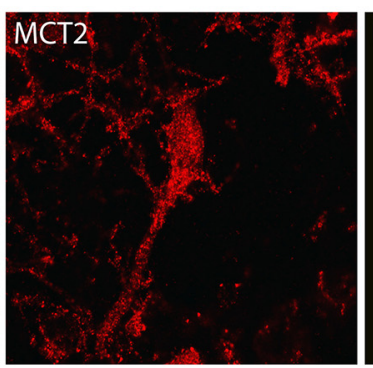

B
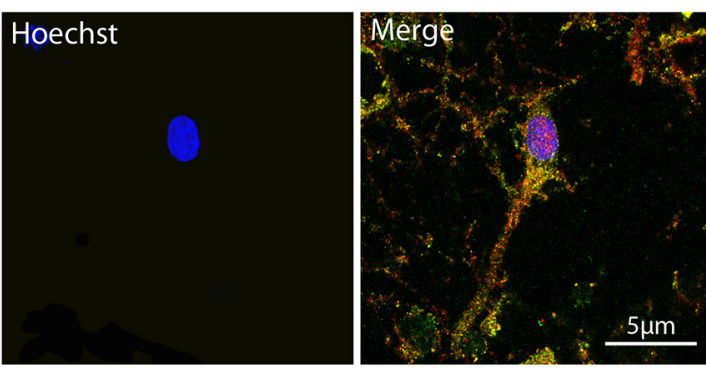

total

protein

extract

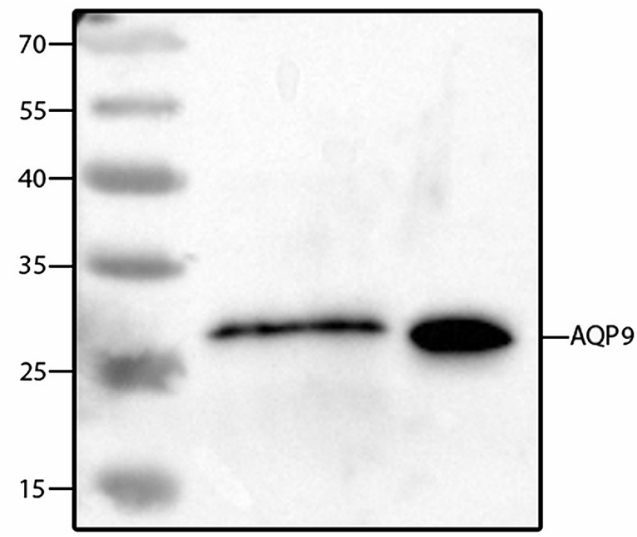

FIGURE 3 | Colocalization and interaction between AQP9 and MCT2 in cultured rat hippocampal neurons (A) Double immunofluorescence labeling for AQP9 (in green) and MCT2 (in red) performed in cultured hippocampal neurons. Colocalization between AQP9 and MCT2 appears as yellow on the merged image (Merge). Nuclei (in blue) were labeled using
Hoechst staining. Immunofluorescence was visualized using confocal microscopy with appropriate filters. (B) Western blot for AQP9 in cultured hippocampal neuron protein extract after immunoprecipitation with anti-MCT2 antibody (IP MCT2) or in total protein extract. This experiment was repeated twice from separate cultures with similar results. 
A

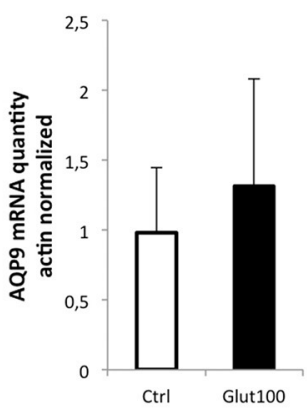

B

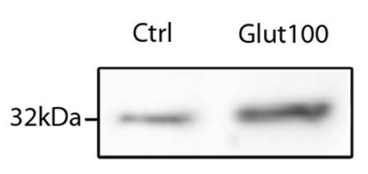

$\beta$-tubulin
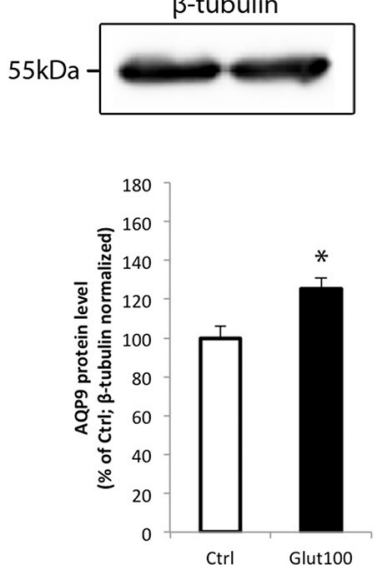

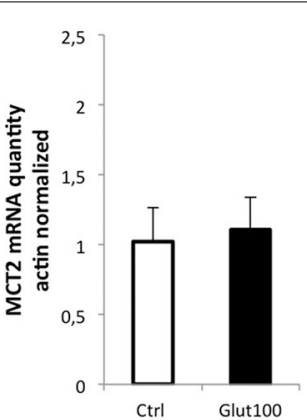

MCT2

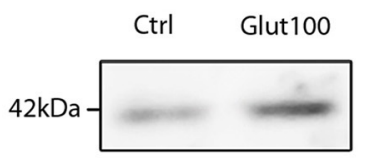

$\beta$-tubulin
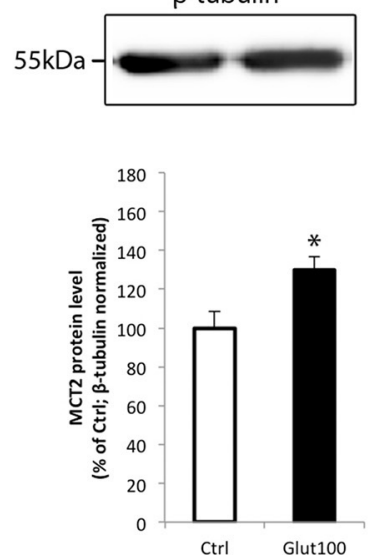

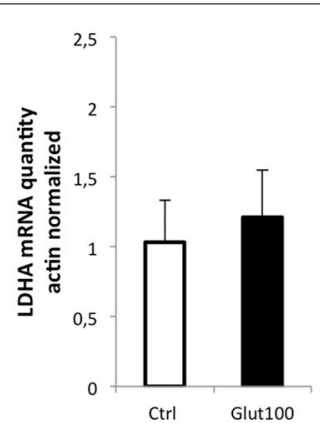

LDHA

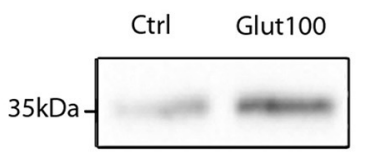

$\beta$-tubulin
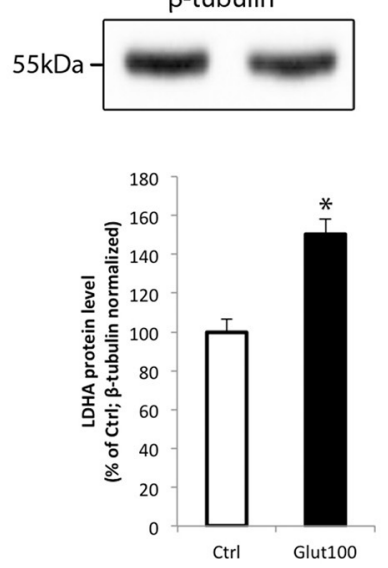

FIGURE 4 | Effect of glutamate on mRNA and protein expression of AQP9, MCT2, and LDHA in cultured rat hippocampal neurons (A) Primary cultures of rat hippocampal neurons were treated with glutamate $100 \mu \mathbf{M}$ for $\mathbf{1 h}$. Then mRNA was extracted and analyzed for AQP9, MCT2 and LDH mRNA expression using RT-PCR. Results are expressed as relative expression vs. control with mean value for control set at 1 after values had been normalized using $\beta$-actin as internal reference. Results represent mean \pm SD with $n=3$. The experiment was repeated three times from different cultures with similar results. Statistical analysis was performed using a non-parametric Mann-Whitney test. No significant difference was observed. (B) Primary cultures of rat hippocampal neurons were treated with glutamate $100 \mu \mathrm{M}$ for $1 \mathrm{~h}$. Then proteins were extracted and analyzed for AQP9, MCT2, and LDH protein expression using Western blot. Blot pictures were obtained with Bio-Rad Chemidoc $^{\text {TM }}$ XRS system (BioRad, Cressier, Switzerland) and quantified using ImageJ software. Quantitative results are expressed as percentage of control after the values had been normalized using $\beta$-tubulin signal as reference. Results represent mean $\pm S D$ with $n=3$. The experiment was repeated three times from different cultures with similar results. Statistical analysis was performed using a non-parametric Mann-Whitney test. An asterisk indicates protein level significantly different from control with $p<0.05$. type. Indeed, it was reported that activation of NMDA receptors (a subtype of glutamate receptors) causes an increase of glucose utilization in cerebellar neurons (Bak et al., 2009). In contrast, exposure of rat hippocampal neurons to glutamate induced a decrease in glucose transport (Porras et al., 2004). Our data showing a decrease in glucose utilization in rat hippocampal neurons after exposure to glutamate are consistent with the study of Porras et al. Although the contradictory results between the finding of Bak et al. on one hand, and ours as well as the study of Porras et al. on the other hand could be explained by the use of a different preparation of neurons (cerebellar vs. hippocampal) as well as by different stimulation conditions (brief NMDA applications in
Mg-free medium for Bak et al. vs. constant glutamate application at $500 \mu \mathrm{M}$ for Porras et al. or $100 \mu \mathrm{M}$ for us), other reasons can be invoked. Thus, it was recently shown that activation of NMDA receptors in rat cortical neurons led to an enhancement of glycolysis at the expense of the pentose phosphate shunt (RodriguezRodriguez et al., 2012). Such an effect, which is due to the stabilization of the enzyme 6-phosphofructo-2-kinase/fructose2,6-bisphosphatase-3 (PFKFB3), a key regulator of glycolysis, eventually causes oxidative stress, and cell death (RodriguezRodriguez et al., 2013). Based on these results, it can be concluded that specific NMDA receptor activation enhances glucose utilization in neurons by activating glycolysis (with the risk of 
inducing cell death later on) while glutamate exposure (which can activate several subtypes of glutamate receptors in parallel) rather leads to reduced glucose utilization by an as yet unknown mechanism.

If we consider that glucose utilization and glycolysis could be reduced upon physiological stimulation with glutamate, then neurons must rely on another energy substrate to face the enhanced energy demands caused by glutamate-dependent depolarization. One possibility could be to use monocarboxylates such as lactate, pyruvate, or the ketone bodies $\beta$-hydroxybutyrate and acetoacetate. Indeed, it was previously shown that neurons preferentially use lactate over glucose-derived pyruvate as preferential oxidative energy substrate, both in vitro (BouzierSore et al., 2003, 2006) and in vivo (Tyson et al., 2003; Serres et al., 2004, 2005; Gallagher et al., 2009; Boumezbeur et al., 2010; Wyss et al., 2011). In order to favor monocarboxylate utilization, specific transporters must be present. It was previously shown that MCT2 is the predominant neuronal monocarboxylate transporter (Bergersen et al., 2001; Pierre et al., 2002). MCT2 was found to be particularly enriched in dendrites, not only at the plasma membrane associated with the postsynaptic density but also in an intracellular pool (Pierre et al., 2009). Moreover, its subcellular distribution was shown to be altered by various stimuli including glutamate, leading to enhanced lactate uptake (Pierre et al., 2009). In parallel, the presence of the aquaglyceroporin AQP9, which can transport small oxidative subtrates such as lactate or glycerol, has been reported in neurons. Thus, its expression was described in some populations of neurons such as catecholaminergic (Badaut et al., 2004) as well as hippocampal and cortical neurons (Mylonakou et al., 2009; Arciénega et al., 2010). The presence of both MCT2 and AQP9 in neuronal mitochondria has been documented. In rat brain sections, MCT2 was found to co-localize with cytochrome oxidase in the inner membrane of neuronal mitochondria in the cortex, the hippocampus and the thalamus (Hashimoto et al., 2008). Similarly, AQP9 was found to be present in brain mitochondrial inner membranes (Amiry-Moghaddam et al., 2005) although the presence of AQP9 in mitochondria has been disputed (Yang et al., 2006). Our results not only confirm that both MCT2 and AQP9 are present in rat hippocampal neuron mitochondria, but they also co-localize and co-immunoprecipitate. These results suggest that both transporters are probably part of a mitochondrial complex that facilitates the entry and use of oxidative substrates. Our observation that glutamate enhances the expression of both MCT2 and AQP9 expression would be consistent with idea that to face enhanced energy demands, neurons would favor the use of extracellular monocarboxylates as oxidative substrates in mitochondria at the expense of glucose. In our case, it could be pyruvate which is present in the culture medium.

Interestingly, the expression of both MCT2 and AQP9 proteins together with the LDHA protein was enhanced after treatment with glutamate, while no change in mRNA expression for all three genes was detected. This observation suggests that a coordinated translational regulation occurs for these three proteins, reinforcing the view that they might form together a complex within mitochondria, as suggested before for MCT2 and LDH (Hashimoto et al., 2008). Previously, it was shown that MCT2 is subject to translational regulation in mouse cortical neurons when treated with different neuroactive substances (Pierre et al., 2003; Chenal and Pellerin, 2007; Chenal et al., 2008; Robinet and Pellerin, 2010). However, it is the first time that both AQP9 and LDHA are shown to undergo a similar regulation in cultured neurons. One possible explanation for the concomitant upregulation of these three mitochondrial proteins by glutamate could be to facilitate the use of oxidative substrates other than glucose (as glucose utilization is decreased under such conditions) in order to face the increased energy demands imposed by excitation. Nevertheless, it remains to be demonstrated whether the oxidation of monocarboxylates and/or glycerol are enhanced under these conditions.

\section{ACKNOWLEDGMENTS}

The authors would like to thank Cendrine Repond for technical assistance as well as Katia Rosafio and Dr. Nadia Steiner for sharing their technical expertise. Fabio Tescarollo was supported by fellowship 10/50349-1 from FAPESP and by Swiss government scholarship $\mathrm{n}^{\circ} 2011.0188$. This work was supported by Swiss National Foundation grant $\mathrm{n}^{\circ} \mathrm{s}$ 31003A-125063 and 31003A-140957 to Luc Pellerin.

\section{REFERENCES}

Amiry-Moghaddam, M., Lindland, H., Zelenin, S., Roberg, B. A., Gundersen, B. B., Petersen, P., et al. (2005). Brain mitochondria contain aquaporin water channels: evidence for the expression of a short AQP9 isoform in the inner mitochondrial membrane. FASEB J. 19, 1459-1467. doi: 10.1096/fj. 04-3515com

Arciénega, I. I., Brunet, J.-F., Bloch, J., and Badaut, J. (2010). Cell locations for AQP1, AQP4 and 9 in the non-human primate brain. Neuroscience 167, 1103-1114. doi: 10.1016/j.neuroscience.2010.02.059

Badaut, J. (2010). Aquaglyceroporin 9 in brain pathologies. Neuroscience 168, 1047-1057. doi: 10.1016/j.neuroscience.2009.10.030

Badaut, J., Brunet, J. F., and Regli, L. (2007). Aquaporins in the brain: from aqueduct to multi-duct. Metab. Brain Dis. 22, 251-263. doi: 10.1007/s11011007-9057-2

Badaut, J., Petit, J.-M., Brunet, J.-F., Magistretti, P. J., Charriaut-Marlangue, C., and Regli, L. (2004). Distribution of Aquaporin 9 in the adult rat brain: preferential expression in catecholaminergic neurons and in glial cells. Neuroscience 128, 27-38. doi: 10.1016/j.neuroscience.2004.05.042

Bak, L. K., Walls, A. B., Schousboe, A., Ring, A., Sonnewald, U., and Waagepetersen, H. S. (2009). Neuronal glucose but not lactate utilization is positively correlated with NMDA-induced neurotransmission and fluctuations in cytosolic $\mathrm{Ca}^{2+}$ levels. J. Neurochem. 109, 87-93. doi: 10.1111/j.1471-4159.2009. 05943.x

Barros, L. F., Courjaret, R., Jakoby, P., Loaiza, A., Lohr, C., and Deitmer, J. W. (2009). Preferential transport and metabolism of glucose in Bergmann glia over Purkinje cells: a multiphoton study of cerebellar slices. Glia 57, 962-970. doi: 10.1002/glia.20820

Bergersen, L., Waerhaug, O., Helm, J., Thomas, M., Laake, P., Davies, A. J., et al. (2001). A novel postsynaptic density protein: the monocarboxylate transporter MCT2 is co-localized with delta-glutamate receptors in postsynaptic densities of parallel fiber-Purkinje cell synapses. Exp. Brain Res. 136, 523-534. doi: $10.1007 / \mathrm{s} 002210000600$

Blair, R. E., Sombati, S., Churn, S. B., and Delorenzo, R. J. (2008). Epileptogenesis causes an $\mathrm{N}$-methyl-d-aspartate receptor/ $\mathrm{Ca}^{2+}$-dependent decrease in $\mathrm{Ca}^{2+} /$ calmodulin-dependent protein kinase II activity in a hippocampal neuronal culture model of spontaneous recurrent epileptiform discharges. Eur. J. Pharmacol. 588, 64-71. doi: 10.1016/j.ejphar.2008. 04.021 
Bliss, T. M., Ip, M., Cheng, E., Minami, M., Pellerin, L., Magistretti, P. J., et al. (2004). Dual-gene, dual-cell type therapy against an excitotoxic insult by bolstering neuroenergetics. J. Neurosci. 24, 6202-6208. doi: 10.1523/JNEUROSCI.0805-04.2004

Boumezbeur, F., Petersen, K. F., Cline, G. W., Mason, G. F., Behar, K. L., Shulman, G. I., et al. (2010). The contribution of blood lactate to brain energy metabolism in humans measured by dynamic 13C nucler magnetic resonance spectroscopy. J. Neurosci. 30, 13983-13991. doi: 10.1523/JNEUROSCI.2040-10.2010

Bouzier-Sore, A.-K., Voisin, P., Bouchaud, V., Bezancon, E., Franconi, J.-M., and Pellerin, L. (2006). Competition between glucose and lactate as oxidative energy substrates in both neurons and astrocytes: a comparative NMR study. Eur. J. Neurosci. 24, 1687-1694. doi: 10.1111/j.1460-9568.2006.05056.x

Bouzier-Sore, A.-K., Voisin, P., Canioni, P., Magistretti, P. J., and Pellerin, L. (2003). Lactate is a preferential oxidative energy substrate over glucose for neurons in culture. J. Cereb. Blood Flow Metab. 23, 1298-1306. doi: 10.1097/01.WCB.0000091761.61714.25

Bradford, M. M. (1976). A rapid and sensitive method for the quantitation of microgram quantities of protein utilizing the principle of protein-dye binding. Anal. Biochem. 72, 248-254. doi: 10.1016/0003-2697(76)90527-3

Chenal, J., and Pellerin, L. (2007). Noradrenaline enhances the expression of the neuronal monocarboxylate transporter MCT2 by translational activation via stimulation of PI3K/Akt and the mTOR/S6K pathway. J. Neurochem. 102, 389-397. doi: 10.1111/j.1471-4159.2007.04495.x

Chenal, J., Pierre, K., and Pellerin, L. (2008). Insulin and IGF-I enhance the expression of the neuronal monocarboxylate transporter MCT2 by translational activation via stimulation of the phosphoinositide 3-kinase-Akt-mammalian target of rapamycin pathway. Eur. J. Neurosci. 27, 53-65. doi: 10.1111/j.14609568.2007.05981.x

Chuquet, J., Quilichini, P., Nimchinsky, E. A., and Buzsaki, G. (2010). Predominant enhancement of glucose uptake in astrocytes versus neurons during activation of the somatosensory cortex. J. Neurosci. 30, 15298-15303. doi: 10.1523/JNEUROSCI.0762-10.2010

Churn, S. B., Anderson, W. W., and DeLorenzo, R. J. (1991). Exposure of hippocampal slices to magnesium-free medium produces epileptiform activity and simultaneously decreases calcium and calcium-dependent protein kinase II activity. Epilepsy Res. 9, 211-217. doi: 10.1016/0920-1211(91) 90054-J

Figley, C. R., and Stroman, P. W. (2011). The role(s) of astrocytes and astrocyte activity in neurometabolism, neurovascular coupling, and the production of neuroimaging signal. Eur. J. Neurosci. 33, 577-588. doi: 10.1111/j.14609568.2010.07584.x

Gallagher, C. N., Carpenter, K. L., Grice, P., Howe, D. J., Mason, A., Timofev, I., et al. (2009). The human brain utilizes lactate via the tricarboxylic acid cycle: a 13C-labelled microdialysis and high-resolution nuclear magnetic resonance study. Brain 132, 2839-2849. doi: 10.1093/brain/awp202

Hartz, A. M., Notenboom, S., and Bauer, B. (2009). Signaling to P-glycoprotein-A new therapeutic target to treat drug-resistant epilepsy? Drug News Perspect. 22, 393-397. doi: 10.1358/dnp.2009.22.7.1401354

Hashimoto, T., Hussien, R., Cho, H. S., Kaufer, D., and Brooks, G. A. (2008). Evidence for the mitochondrial lactate oxidation complex in rat neurons: demonstration of an essential component of brain lactate shuttles. PLoS ONE 3:e2915. doi: 10.1371/journal.pone.0002915

Heid, C. A., Stevens, J., Livak, K. J., and Williams, P. M. (1996). Real time quantitative PCR. Genome Res. 6, 986-994. doi: 10.1101/gr.6.10.986

Jakoby, P., Schmidt, E., Ruminot, I., Gutiérrez, R., Barros, L. F., and Deitmer, J. W. (2014). Higher transport and metabolism of glucose in astrocytes compared with neurons: a multiphoton study of hippocampal and cerebellar tissue slices. Cereb. Cortex 24, 222-231. doi: 10.1093/cercor/bhs309

Loaiza, A., Porras, O. H., and Barros, L. F. (2003). Glutamate triggers rapid glucose transport stimulation in astrocytes as evidenced by real-time confocal microscopy. J. Neurosci. 23, 7337-7342.

Magistretti, P. J. (1999). "Brain energy metabolism," in Fundamental Neuroscience, eds M. J. Zigmond, F. E. Bloom, S. C. Landis, J. L. Roberts, and L. R. Squire (San Diego, CA: Academic Press), 389-413.

Mylonakou, M. N., Petersen, P. H., Rinvik, E., Rojek, A., Valdimarsdottir, E., Zelenin, S., et al. (2009). Analysis of mice with targeted deletion of AQP9 gene provides conclusive evidence for expression of AQP9 in neurons. J. Neurosci. Res. 87, 1310-1322. doi: 10.1002/jnr.21952
Pellerin, L. (2010). Food for thought: the importance of glucose and other energy substrates for sustaining brain function under varying levels of activity. Diabetes Metab. 36, S59-S63. doi: 10.1016/S1262-3636(10) 70469-9

Pellerin, L., and Magistretti, P. J. (1994). Glutamate uptake into astrocytes stimulates aerobic glycolysis: a mechanism coupling neuronal activity to glucose utilization. Proc. Natl. Acad. Sci. U.S.A. 91, 10625-10629. doi: 10.1073/pnas.91.22.10625

Pellerin, L., and Magistretti, P. J. (1996a). Excitatory amino acids stimulate aerobic glycolysis in astrocytes via an activation of the $\mathrm{Na}^{+} / \mathrm{K}^{+}$ATPase. Dev. Neurosci. 18, 336-342. doi: 10.1159/000111426

Pellerin, L., and Magistretti, P. J. (1996b). The contribution of astrocytes to the 18F-2-deoxyglucose signal in PET activation studies. Mol. Psychiatry 1, 445-452.

Pellerin, L., and Magistretti, P. J. (2012). Sweet sixteen for ANLS. J. Cereb. Blood Flow Metab. 32, 1152-1166. doi: 10.1038/jcbfm.2011.149

Pierre, K., Chatton, J. Y., Parent, A., Repond, C., Gardoni, F., Di Luca, M., et al. (2009). Linking supply to demand: the neuronal monocarboxylate transporter MCT2 and the alpha-amino-3-hydroxyl-5-methyl-4-isoxazolepropionic acid receptor GluR2/3 subunit are associated in a common trafficking process. Eur. J. Neurosci. 29, 1951-1963. doi: 10.1111/j.1460-9568.2009. 06756.x

Pierre, K., Debernardi, R., Magistretti, P. J., and Pellerin, L. (2003). Noradrenaline enhances monocarboxylate transporter 2 expression in cultured mouse cortical neurons via a translational regulation. J. Neurochem. 86, 1468-1476. doi: 10.1046/j.1471-4159.2003.01964.x

Pierre, K., Magistretti, P. J., and Pellerin, L. (2002). MCT2 is a major neuronal monocarboxylate transporter in the adult mouse brain. J. Cereb. Blood Flow Metab. 22, 586-595. doi: 10.1097/00004647-200205000-00010

Pierre, K., and Pellerin, L. (2005). Monocarboxylate transporters in the central nervous system: distribution, regulation and function. J. Neurochem. 94, 1-14. doi: 10.1111/j.1471-4159.2005.03168.x

Pierre, K., Pellerin, L., Debernardi, R., Riederer, B. M., and Magistretti, P. J. (2000). Cell-specific localization of monocarboxylate transporters, MCT1 and MCT2, in the adult mouse brain revealed by double immunohistochemical labeling and confocal microscopy. Neuroscience 100, 617-627. doi: 10.1016/S03064522(00)00294-3

Porras, O. H., Loaiza, A., and Barros, L. F. (2004). Glutamate mediates acute glucose transport inhibition in hippocampal neurons. J. Neurosci. 24, 9669-9673. doi: 10.1523/JNEUROSCI.1882-04.2004

Robinet, C., and Pellerin, L. (2010). Brain-derived neurotrophic factor enhances the expression of the monocarboxylate transporter 2 through translational activation in mouse cultured cortical neurons. J. Cereb. Blood Flow Metab. 30, 286-298. doi: 10.1038/jcbfm.2009.208

Rodriguez-Rodriguez, P., Almeida, A., and Bolanos, J. P. (2013). Brain energy metabolism in glutamate-receptor activation and excitotoxicity: role for APC/C-Cdh1 in the balance glycolysis/pentose phosphate pathway. Neurochem. Int. 62, 750-756. doi: 10.1016/j.neuint.2013.02.005

Rodriguez-Rodriguez, P., Fernandez, E., Almeida, A., and Bolanos, J. P. (2012). Excitotoxic stimulus stabilizes PFKFB3 causing pentose-phosphate pathway to glycolysis switch and neurodegeneration. Cell Death Differ. 19, 1582-1589. doi: 10.1038/cdd.2012.33

Serres, S., Bezancon, E., Franconi, J. M., and Merle, M. (2004). Ex vivo analysis of lactate and glucose metabolism in the rat brain under different states of depressed activity. J. Biol. Chem. 279, 47881-47889. doi: 10.1074/jbc.M409429200

Serres, S., Bezancon, E., Franconi, J. M., and Merle, M. (2005). Ex vivo NMR study of lactate metabolism in rat brain under various depressed states. J. Neurosci. Res. 79, 19-25. doi: 10.1002/jnr.20277

Sokoloff, L. (1993). Sites and mechanisms of function-related changes in energy metabolism in the nervous system. Dev. Neurosci. 15, 194-206. doi: $10.1159 / 000111335$

Tyson, R. L., Gallagher, C., and Sutherland, G. R. (2003). 13C-Labeled substrates and the cerebral metabolic compartmentalization of acetate and lactate. Brain Res. 992, 43-52. doi: 10.1016/j.brainres.2003.08.027

Wyss, M. T., Jolivet, R., Buck, A., Magistretti, P. J., and Weber, B. (2011). In vivo evidence for lactate as a neuronal energy source. J. Neurosci. 31, 7477-7485. doi: 10.1523/JNEUROSCI.0415-11.2011 
Yang, B., Zhao, D., and Verkman, A. S. (2006). Evidence against functionally significant aquaporin expression in mitochondria. J. Biol. Chem. 281, 16202-16206. doi: 10.1074/jbc.M601864200

Conflict of Interest Statement: The authors declare that the research was conducted in the absence of any commercial or financial relationships that could be construed as a potential conflict of interest.

Received: 23 May 2014; accepted: 23 July 2014; published online: 12 August 2014. Citation: Tescarollo F, Covolan L and Pellerin L (2014) Glutamate reduces glucose utilization while concomitantly enhancing AQP9 and MCT2 expression in cultured rat hippocampal neurons. Front. Neurosci. 8:246. doi: 10.3389/fnins. 2014.00246

This article was submitted to Neuroenergetics, Nutrition and Brain Health, a section of the journal Frontiers in Neuroscience.

Copyright (c) 2014 Tescarollo, Covolan and Pellerin. This is an open-access article distributed under the terms of the Creative Commons Attribution License (CC BY). The use, distribution or reproduction in other forums is permitted, provided the original author(s) or licensor are credited and that the original publication in this journal is cited, in accordance with accepted academic practice. No use, distribution or reproduction is permitted which does not comply with these terms. 